Universidad de Lima

Facultad de Comunicación

Carrera de Comunicación

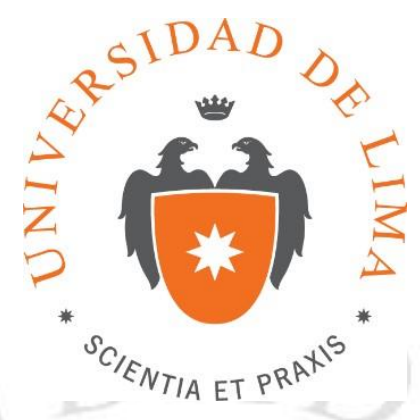

\title{
ANÁLISIS SEMIÓTICO DEL CURSO DE RELIGIÓN EN COLEGIOS DE DISTINTAS CONFESIONES
}

Trabajo de investigación para optar la licenciatura en Comunicación

Alonso Bracamonte Novella

Código 20041563

Asesor

Lilian Kanashiro Nakahodo

Lima - Perú

Julio 2016 


\section{ANÁLISIS SEMIÓTICO DEL CURSO DE RELIGIÓN EN COLEGIOS DE DISTINTAS}

CONFESIONES 


\section{RESUMEN}

El siguiente estudio de investigación tiene como objetivo principal analizar las prácticas semióticas que se activan en los procesos del curso de religión en un colegio evangélico, uno católico y uno laico y compararlos entre sí.

Algunas teorías y estudios anteriores tienen relación con los resultados encontrados en este proyecto, pero con ciertas diferencias encontradas en el caso del Perú y la muestra observada (tres colegios de diferentes confesiones de la ciudad de Lima). Empleamos como metodología de análisis el punto de vista semiótico, y hacemos una comparación entre las prácticas, estrategias y formas de vida, las cuales guardan tanto semejanzas como diferencias entre los colegios de la muestra analizada.

PALABRAS CLAVE: religión, educación, semiótica, prácticas, estrategias, formas de vida. 


\section{INTRODUCCIÓN}

Existen diferentes maneras de relacionar la educación y la religión. Una puede ser la pedagogía y cómo es aplicada. También el desenvolvimiento de los profesores, así como su relación con los alumnos hasta la obligatoriedad o participación del gobierno en la enseñanza de religión. La enseñanza de religión abarca muchos aspectos, como el grado en que los estudiantes la aplican en su vida y hasta qué punto respetan las normas impuestas por esta, pasando por los aspectos que cambian de país a país y el estudio de contextos más cercanos, como el latinoamericano.

El siguiente estudio de investigación tiene como objetivo principal analizar las prácticas semióticas que se activan en los procesos del curso de religión en un colegio evangélico, uno católico y uno laico y compararlos entre sí. Para ello empleamos los conceptos de prácticas, estrategias y formas de vida, conceptos acuñados por Jacques Fontanille. Asimismo, tomamos como referencia los estudios de Jean Marie Floch y Eric Landowski.

\section{ESTUDIOS ACTUALES SOBRE RELIGIÓN EN EDUCACIÓN}

Diversos autores abordan este tema como, por ejemplo, Siebzehner y Lehmann, quienes estudian el surgimiento de una nueva corriente educacional étnicoreligiosa en Israel: el Maayan Hachinuch Hatorani ("El manantial de la educación de la Torah"), de identidad sefaradí-oriental, contraria a la ya establecida educación y a las políticas de discriminación implementadas en las instituciones asquenazíes ultraortodoxas (Siebzehner y Lehmann 2008). Nanbu Hirotaka, por su parte, estudia cómo en sus inicios el gobierno chino veía la religión como un enemigo del sistema hasta que, a partir de 1970 hasta la fecha, empezó a convertirse en un aliado de las autoridades (Nanbu 2008).

En el caso específico de Latinoamérica y la educación católica, tenemos el estudio realizado por Torrendell, quien enfoca, desde un punto de vista católico, de las tendencias y problemas culturales, educativos y magisteriales de la educación católica en Sudamérica, considerando aspectos tales como: el monopolio o unanimidad de la educación católica en la época colonial, las tensiones entre el Estado y la educación 
católica durante las primeras décadas posteriores a las independencias de los países latinoamericanos y la consolidación del Estado educador y el crecimiento paralelo de la educación (Torrendell 2010).

Siguiendo con los antecedentes, tenemos a Gärtner y Könemann, quienes tratan el tema de lo que sucede cuando la religión llega al colegio y ambos entran en contacto. Trata ambos lados: las consecuencias para la institución religiosa y parroquias cristianas, así como para los colegios y estudiantes. Según este estudio, en las regiones de habla alemana, la religión ha sido parte del plan de estudios de la escuela durante muchos siglos. Hasta el presente, la escuela era, entre otras cosas, un lugar para el aprendizaje de la religión. Debido a la ruptura con la tradición cristiana y el resultante debilitamiento asociado de los lugares tradicionales de aprendizaje, tales como la familia y la parroquia, la escuela cada vez más se convierte en el lugar predominante para la educación religiosa y la socialización (Gärtner y Könemann 2013). En la misma línea, tenemos a Sharma y Guest, quienes investigan cómo viven su identidad cristiana los estudiantes universitarios practicantes; además, toman nota de los detalles de esta experiencia y los diferentes casos, como juntarse con estudiantes iguales a ellos o, en otros, dejar de lado sus creencias al encontrarse en un ambiente diferente como la universidad (Sharma y Guest 2013).

Por su parte, Schwadel busca negar la afirmación de que la educación escolar merma o disminuye las creencias religiosas de los estudiantes en EEUU. Según este autor, si bien algunas de estas son mermadas, otras se ven agudizadas. Por ejemplo, según su investigación, la educación escolar efectivamente hace disminuir las creencias literales en la Biblia y los puntos de vista exclusivamente religiosos; sin embargo, no afecta en lo absoluto otras creencias religiosas, como la existencia de Dios o la vida después de la muerte. Asimismo, sostiene que la educación agudiza ciertas prácticas, como la participación en actividades religiosas, actividades devocionales y la enfatización de la religión en la vida diaria. Según el autor, la educación en EEUU ciertamente tiene efectos sobre las creencias religiosas, pero estos son complejos (Schwadel 2011).

La mayoría de investigaciones actuales analizan los diferentes impactos de la educación religiosa y las reacciones de los estudiantes, tanto laicos como cristianos, ante esta. Por ejemplo, Segall y Burke buscan demostrar que, a pesar de que en la mayoría de los casos la religión no forma parte de la currícula escolar de EEUU, se 
encuentra de manera implícita en muchas formas. Según los investigadores, la visión judeo-cristiana del mundo continúa teniendo influencia en la educación pública de los EEUU. Buscan mostrar cómo la Biblia continúa siendo un texto con autoridad, incluso en los ambientes académicos (Segall y Burke 2013). Todos estos estudios aportan a la investigación en cuanto a la comparación con el caso peruano y la muestra estudiada, lo cual analizaremos más adelante.

\section{HACIA UNA SEMIÓTICA DE LA EDUCACIÓN RELIGIOSA}

Como sabemos, nuestra investigación se basa en el análisis comparativo del curso de religión en tres colegios de confesiones distintas (evangélico, católico y laico). Para poder realizar este análisis, nos enfocaremos desde el punto de vista semiótico, del cual usaremos como elemento de análisis los planos de inmanencia. Existen al menos seis planos de inmanencia; sin embargo, nos vamos a focalizar en tres: las prácticas, las estrategias y las formas de vida.

Dándole una breve revisión a la teoría sobre los planos de inmanencia, podemos empezar mencionando que se trata de un modelo creado por el semiótico francés Jacques Fontanille. Los planos de inmanencia son una forma de entender el proceso de significación desde el plano de la expresión como la presencia constante de seis planos (las formas de vida, las estrategias, la práctica, el objeto, el texto y los signos). No obstante, la inmanencia de los planos está sujeta a una jerarquía y compuesta a su vez por interfaces. Cada plano posee dos interfaces: una faz "textual", que vendría a ser la forma; y una faz "práxica", que vendría a ser la parte sustancial o material, es decir, el "soporte material"correspondientes a la sustancia y forma. Asimismo, los planos de inmanencia del plano de la expresión no pierden su correlación propioceptiva con el plano del contenido (Fontanille, 2014).

\section{a. Las prácticas}

Las prácticas consisten en cursos de acción, principalmente definidos por el tema tratado y por los diferentes roles que este exige para que la acción tenga lugar. Es la forma sintagmática que permite acoger conjuntamente $\mathrm{y}$ de manera congruente signos, textos y objetos, al mismo tiempo que la práctica misma. También mira hacia los niveles superiores: estrategias y formas de vida. Es la materia sobre la cual se 
apoyan las estrategias, las cuales están ubicadas en un nivel superior (Fontanille, 2013 y 2014). En ese sentido, consideramos que una clase de religión o de cualquier material supondría una práctica que presume la existencia de dos roles, el educador bajo el rol de la enseñanza y el educando bajo el rol del aprendizaje. Cada uno productor de textos y signos, aproximaciones sobre temas diversas como la creencia religiosa, su aplicación o teorización sobre la vida cotidiana. Entre ello cabe mencionar la existencia de textos y signos bajo el soporte del objeto sagrado, como por ejemplo los libros de la doctrina, los símbolos y gestos religiosos por excelencia así como las oraciones o rezos que adquieren valoraciones especiales que a nivel hipotético consideramos estarán presentes en los cursos en donde realizaremos la observación.

Resulta sugerente vincular el concepto de práctica esbozando anteriormente con los regímenes de interacción propuestos por Landowski en su estudio sobre el riesgo. El autor define cuatro regímenes como los más importantes: programación, asentimiento, manipulación y ajuste. La programación se basa en el principio de regularidad o rol temático de un objeto o personaje que, previamente programado, actúa de acuerdo a ese programa, de manera similar a una máquina (por ejemplo, un policía que siempre reacciona de la misma manera). El régimen del asentimiento, que en primeras versiones fue denominada como accidente, está basado en el azar o probabilidad, pudiendo ser de dos tipos matemática o mítica. La manipulación está basado en la intencionalidad del manipulador y en el hacer-ser, hacer-hacer o hacerquerer. Según el autor, dentro de este régimen se encuentran las competencias modales. La manipulación se guía por el principio de motivación estableciendo dos tipos: consensual (hacer-querer) y decisional, que apela al "deber hacer". Por último, tenemos el régimen del ajuste, que se basa en la sensibilidad y en la competencia estésica. En el ajuste, encontramos la sensibilidad perceptiva que nos permite no solamente experimentar por los sentidos las variaciones perceptibles del mundo exterior (ligadas a la presencia de otros cuerpos/sujetos o los elementos del mundo/objeto) y sentir las modulaciones internas que afectan los estados del cuerpo propio; sino también interpretar el conjunto de esas soluciones de continuidad en términos de sensaciones diferenciadas que por sí misma hacen sentido. El otro tipo de sensibilidad, presente en el régimen del ajuste, es la sensibilidad reactiva, que es aquella que se le atribuye, por ejemplo, a objetos cuando decimos que estos son "sensibles", los cuales son elementos 
sin vida y que no "sienten" realmente, pero sí responden a los impulsos mecánicos, eléctricos u otros, a los que los sometemos (Landowski 2009).

La conjunción planteada resulta pertinente tomando en cuenta un elemento común que une a ambas aproximaciones teóricas: las competencias modales. La tipología de prácticas elaboradas por Fontanille en su acercamiento a la optimización (praxis, procedimiento, conducta, protocolo, ritual autónomo y heterónomo) se construyen a partir de una combinatoria de modalidades, las mismas empleadas para la tipificación de las interacciones arriesgadas de Landowski. Si la enseñanza es considerada una práctica esta puede ser planteada como un procedimiento, protocolo o conducta dependiendo de los signos, textos y objetos puestos en escena por el sujeto de enunciador de la práctica y modalizadores de la misma. Pero al mismo tiempo y tomando en cuenta la interacción con el sujeto enunciatario de la práctica enseñanza, podemos considerar que dicha práctica está basada en una programación, manipulación, ajuste o asentimiento. Una u otra dependenderán de las competencias manifestadas en las prácticas.

\section{b. Las estrategias}

Cada escena práctica debe acomodarse, en el espacio y en el tiempo, a las otras escenas y prácticas, concomitantes o no concomitantes. La estrategia es, en suma, un principio de composición sintagmática de las prácticas entre sí. Una definición sencilla sería decir que la interacción de varias prácticas forma una estrategia. Las estrategias aportan específicamente un "horizonte" de valores dominantes, en nombre de los cuales las prácticas son ordenadas y dispuestas entre sí. En ese sentido, al abordar las prácticas de enseñanza en los tres colegios de confesiones religiosas diferentes supone observar cómo las prácticas se combinan y superponen para constituir estrategias. Este proceso sintagmático es creador de sentido, más allá de la que aportan las prácticas en sí mismas. Al interior del plano correspondiente a las estrategias, Fontanille nos plantea la distinción entre la gestión estratégica de las prácticas y la iconización de los comportamientos estratégicos. En el primero, correspondiente a la faz "formal" de las estrategias encontramos las formas de control de los procesos de acomodación y en la faz "sustancial" la creación de estilos estratégicos entendidos como comportamientos (Fontanille, 2014). 
Tomando en cuenta la estilística que las estrategias pueden tomar, Fontanille sugiere apoyarse en los estudios de Jean Marie Floch y las nociones de continuidad y discontinuidad, en su célebre análisis sobre los viajeros del tren (1993), quien las organiza de acuerdo a su flujo de continuidad, discontinuidad, no continuidad y no discontinuidad:

Discontinuidad

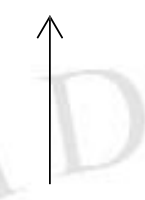

No continuidad

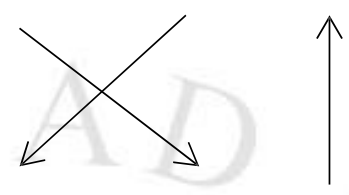

No discontinuidad

Los elementos de las estrategias pueden impulsar la creación de continuidad o discontinuidad. Cuando tienen una estrategia de creación de continuidad —es decir, que están compuestos por una serie de comprensiones de continuidades-, se dejan llevar por el flujo y no hacen caso de las marcas, de los límites, de los confines, no reaccionan ante los momentos álgidos del recorrido. Por otro lado, en el extremo opuesto se encuentra la creación de discontinuidad, en la que los elementos de la estrategia crean una discontinuidad, manifiestan una cierta sensibilidad a los juegos de identidad y alteridad. Entre los dos, se ubica los elementos de no continuidad y no discontinuidad.

Para ilustrar un ejemplo, Floch utiliza a viajeros del metro clasificándolos como "agrimensores", aquellos que valorizan la discontinuidad; "sonámbulos", quienes valorizan las continuidades; "callejeros", los que son ejemplo de las no-continuidades; y "dinámicos", que valorizan las no discontinuidades. Estos cuatros tipo de viajeros tienen características diferentes: los primeros buscan y aprecian los trayectos discontinuos como "recorridos", los dinámicos realizan o intentan realizar "encadenamientos" (es decir, performances), los sonámbulos son los viajeros de la continuidad y realizan "trayectorias" y, por último, los callejeros son los aficionados los "paseos" (Floch 1993: 38-65).

Este ejemplo y categorización de Floch es útil en esta investigación porque, si bien este trabajo se trata sobre el curso de religión en colegios de diferentes confesiones, estos adquieren en sus estrategias comportamientos de continuidad y discontinuidad y pueden ser llamados metafóricamente tanto "agrimensores" en unos 
casos como "dinámicos" en otros. De manera hipotética, podemos imaginar profesores que calculan cada elemento de la clase y la segmentan con especial cuidado y al otro extremo, docentes que repiten a ojos cerrados sin mayor motivación al modo de los sonámbulos. Las negaciones de la tipología (dinámicos y callejeros) también es posible de encarnarse en los trayectos pedagógicos de los profesores. Hay quienes guardan expectativas por lo que el alumnos pueden ofrecer a partir de los estímulos metodológicos y hay que quienes buscan producir encadenamientos creativos entre lo que plantea la doctrina y los retos de la vida mundana.

\section{c. Las formas de vida}

Las formas de vida forman un horizonte cultural de comportamiento constante que tiende a repetirse de manera constante a lo largo del tiempo a menos que cambie el contexto y el fenómeno observado. Diferentes estrategias y prácticas pueden llevar a la misma forma de vida así como diferir de esta.

"Es el nivel más alto de los planos de inmanencia. La experiencia subyacente, el sentimiento de una identidad de comportamiento, la percepción de una regularidad en el conjunto de procedimientos de acomodación estratégica es la experiencia de un ethos; dicha experiencia, al convertirse en un dispositivo de expresión pertinente (un estilo que expresa una actitud), da lugar a una forma de vida susceptible de integrar la totalidad de los niveles inferiores para producir globalmente una configuración pertinente para el análisis de las cultura" (Fontanille, 2008).

En ese sentido, la organización práxica y estratégica nos lleva preguntarnos si lo que encontramos en cada colegio responde realmente a diferencias o a una semejanza que las une de forma subyacente. ¿No es la religión una forma de vida que se ha desplegado en formas de expresión aparentemente diferentes? O contrariamente, cada forma de expresión religiosa nos lleva a formas de vida diferentes, y cada una de ellas se conecta con expresiones estratégicas y prácticas en otras áreas de la vida política, social y económica. En ese sentido, encontraríamos en las formas de vida religiosa la organización de diferentes formas de organización de la cultura que entran en pugna o cohabitan tensamente en nuestro mundo.

Finalmente las formas de vida, podrían tomarse tanto como el plano más alto o como el más bajo de los planos de inmanencia. Los puntos de partida y final son arbitrarios y dependen en gran parte de la naturaleza de la investigación. Tal es el caso de nuestra investigación ni tomó el punto de partida más bajo ni el más alto en cualquier 
punto de vista que se quiera tomar. Nuestra opción metodológica ha sido ingresar al análisis por el punto medio, por el plano de las escenas prácticas para arribar y responder a nuestra interrogante sobre las formas de vida. Creemos firmemente que la educación religiosa entendida como práctica de enseñanza -sin desdeñar la importancia de las prácticas de aprendizaje- activa procesos semióticos que nos acercan a la organización e hipotética cohesión del mundo cultural en que vivimos y puede brindarnos pistas oportunas para comprender la complejidad del mundo actual considerando el trasfondo histórico colonial que nos antecede.

\section{METODOLOGÍA}

Para esta investigación hemos seleccionado el método conocido como “observación externa o no participante". Este modelo hace referencia a cuando el observador extrae sus datos sin una participación en los acontecimientos de la vida del grupo que estudia (Pardinas 1978). Una de las ventajas de la observación no participante es el hecho de que el investigador puede dedicar toda su atención a la observación y a realizar las anotaciones, al mismo tiempo que transcurren los fenómenos y permite también recoger datos para la comprobación empírica de las hipótesis (Anguera 1997).

Para el análisis y comparación de los procesos semióticos reconocibles dentro de las instituciones escolares, que fueron nuestra muestra, se empezó, antes que todo, contactando con estos para solicitar el permiso de introducir un observador dentro de las aulas de clase. Para ello hubo que hacer llamadas y recurrir a contactos personales que tuvieran algún tipo de relación con las autoridades de las respectivas instituciones. Las respuestas no fueron las mismas en cada una de las instituciones. Por citar un ejemplo, en el caso del colegio laico (Independencia, en Miraflores), la directora aceptó sin mayor reparo la observación de tres clases de religión de diferentes grados (cuarto, quinto y sexto de primaria) e indicó determinadas fechas en las que debía acercarme a observar las clases. Sin embargo, llegada la fecha pactada no se pudo realizar la observación debido a la resistencia de la profesora de religión (la cual era la misma para 
las tres secciones) y de la coordinadora. Ante la ausencia de la directora en ese momento, no quedó otra alternativa que postergar la observación. Finalmente, después de hacerle saber a la directora del colegio sobre lo sucedido, se programaron las observaciones para tres semanas después de la fecha indicada. Se logró con esto, a pesar de la resistencia del personal, ingresar a las aulas de clase a observar las secciones respectivas.

En el caso del colegio católico (San José de Cluny, en Barranco), se encontró una fuerte resistencia desde el comienzo por parte de la directora y las autoridades no eclesiásticas del colegio. A través de contactos personales, se pudo llegar a contactar con la pastoral del colegio, que es el grupo conformado por religiosas y personal de cargo eclesiástico encargado del programa de religión. Al explicarles el objetivo de nuestra investigación, aceptaron las mismas condiciones mencionadas anteriormente en el colegio laico; sin embargo, en este caso sí cumplieron con las fechas pactadas y el trato fue amable una vez pasada la resistencia inicial.

Finalmente, el colegio evangélico (Jesús Salvador, en Surco), al ser contactado, se arregló una cita por la directora, quien me habló del modo de vida que se trataba de impartir en el colegio y que en su caso ellos sí estaban interesados en darlo a conocer. Se pactaron las fechas y se cumplieron sin mayor problema.

Una vez empezada la investigación de campo y la observación de diferentes indicadores dentro de las aulas de clase, citamos algunos ejemplos:

- La duración de las clases.

- La forma en que los profesores dictaban la clase (tono de voz, gestualización, etc.).

- Elementos que utilizaba el profesor, sean imágenes, video, películas, audio, etc.

- Temas tratados.

- Estrategias del profesor para captar la atención de sus alumnos.

- Distancia entre el profesor y el alumno (es decir, se nota una fuerte autoridad y verticalidad o la relación es más horizontal). 
Entre los instrumentos utilizados, se recurrió a la grabación en audio de las clases con una pequeña grabadora portátil y al apunte de notas en un cuaderno mientras se dictaba la clase. Mi ubicación como investigador era en un pupitre al fondo del salón.

La técnica era básicamente anotar todo indicador que ayudase a determinar qué prácticas o estrategias se estaban utilizando, para deducir después las formas de vida de cada uno de los colegios de la muestra. En este caso, la observación no participante fue la mejor opción; ya que, en la mayoría de los casos, no se despertó la atención de los alumnos como observador debido a mi ubicación al fondo de la clase ni se participó de modo alguno en las actividades realizadas durante el dictado. De esta manera, se pudo observar cómo se llevaba a cabo la clase sin que mi intervención genere algún tipo de cambio.

Se realizaron nueve visitas, tres por cada colegio seleccionado, divididas en tres aulas: cuarto, quinto y sexto grado de primaria. El tiempo de observación de cada clase fue de aproximadamente dos horas, dando un total de 18 horas de observación. Los datos recogidos se consignaron en un diario de campo por cada colegio, permitiendo llevar a cabo las comparaciones respectivas, distinguiendo las semejanzas y diferencias. Luego de ello y con la información ordenada según los indicadores, procedimos a trabajar el análisis propiamente semiótico, cuidando en todo momento de analizar las situaciones observadas y neutralizando en lo que fuera posible las subjetivaciones del observador. A continuación presentamos los hallazgos encontrados a partir de la observación y el análisis semiótico.

\section{RESULTADOS}

Señalamos anteriormente nuestra opción metodológica de ingresar al análisis semiótico por el punto medio: las prácticas semióticas, acorde al objetivo general planteado para la presente investigación. En ese sentido, hemos sostenido a modo de hipótesis que la educación religiosa entendida como práctica de enseñanza activa procesos semióticos que nos acercan a la cohesión del mundo cultural bajo una forma de vida que trasciende las peculiaridades religiosas o más bien nos plantea el camino 
para comprender la complejidad del mundo actual en diferentes formas de vida. Organizamos los hallazgos en el orden planteado previamente en nuestros objetivos específicos:

\section{a. Prácticas}

Siguiendo los objetivos específicos de la investigación - los cuales fueron, desde un inicio, descubrir a través de la observación no participante las prácticas, estrategias y formas de vida de tres colegios de diferentes confesiones de la ciudad de Lima-, vamos a empezar por mencionar algunas semejanzas y diferencias de las prácticas semióticas principalmente definidos por el tema de la acción en curso y por los diferentes roles que ese tema exige para que la acción tenga lugar.

Analizando las semejanzas y diferencias entre los diferentes colegios, encontramos, entre las prácticas, aquella de persignarse antes de empezar clases, la cual es realizada en el colegio católico (San José de Cluny) y el laico (Independencia). Esta práctica difiere de lo hallado en el colegio evangélico (Jesús Salvador), en el cual no se persignan; sin embargo, esto puede definirse como una semejanza en el sentido de que los tres colegios siguen ritos gestuales de acuerdo a su confesión, ya que el acto de persignarse no es practicado en la religión evangélica.

Como diferencia, encontramos que en algunos casos, en el colegio católico (San José de Cluny), se coloca una religiosa con cargo eclesiástico como profesora, mientras que en el evangélico (Jesús Salvador) se coloca una profesora practicante de la religión evangélica, pero sin cargo eclesiástico -que en el caso de la religión evangélica sería un pastor-.

También encontramos como diferencias en las prácticas que la religiosa y profesora del colegio católico (San José de Cluny) de una de las secciones utiliza un tono muy bajo de voz y no demuestra algún signo indicador de autoridad fuerte, mientras que en el colegio evangélico (Jesús Salvador) la profesora alza mucho la voz y gesticula bastante al igual que en el colegio laico (Independencia).

Otra semejanza encontrada en las prácticas es el uso de libros que van de acuerdo a la confesión de cada colegio. En el caso del colegio católico (San José de Cluny), estudian pasajes sacados directamente de la Biblia; en el evangélico (Jesús 
Salvador), utilizan un libro especial de educación cristiana; y en el laico (Independencia), uno de confesión católica, el cual es utilizado para cumplir con la currícula que manda el Ministerio de Educación para el curso de religión.

Volviendo al tema de las oraciones, observamos que la práctica de la oración del Ave María es recurrente en todas las secciones estudiadas en el colegio católico (San José de Cluny), mientras no lo es en el evangélico (Jesús Salvador); lo que puede señalarse como una semejanza entre ambos colegios en la coherencia con sus respectivos ritos verbales (rezos).

Una diferencia en las prácticas analizadas es que, tanto en las secciones estudiadas del colegio católico como del laico (San José de Cluny e Independencia), la profesora dicta la clase en forma apurada y sin ponerle mucha emoción a la clase; mientras que en el colegio evangélico (Jesús Salvador), la profesora le pone mucha intensidad a su forma de dictar la clase, incluso llegando a hacer ella misma pequeñas representaciones de los pasajes de la Biblia. Otra diferencia que llama particularmente la atención es el trato de los hombres y las mujeres, el cual difiere en las prácticas realizadas por el colegio católico y laico (San José de Cluny e Independencia), donde tratan por igual a ambos sexos; mientras que, en el colegio cristiano (Jesús Salvador), hay un trato más estricto hacia los alumnos hombres en general, a los cuales se les dice que deben cumplir con su rol de jefes del hogar en el futuro.

Asimismo en el ámbito de las prácticas diferenciada tenemos los temas planteados en clase. En el caso de los colegios católico y laico (San José de Cluny e Independencia), son básicamente históricos y, puede decirse, pasajes clásicos de la Biblia; mientras que, en el evangélico (Jesús Salvador), se mencionan temas polémicos, como la fornicación y el adulterio, con la finalidad de atraer la atención de los alumnos. Una práctica encontrada solo en el colegio católico (San José de Cluny) consiste en que allí llamaban "visitas misioneras" al hecho de ir a visitar otros colegios (en este caso, estatales) para hablarles sobre la importancia de Dios y la religión católica en sus vidas. Esta práctica no se halló ni en el colegio evangélico ni en el laico. También encontramos, en el ámbito de las prácticas, que los niños suelen distraerse casi o igual en los tres colegios, para lo cual los profesores utilizan diferentes estrategias que veremos más adelante. 


\section{b. Estrategias}

Señalábamos que la combinación de prácticas trae como consecuencia la estrategia. Para ser más precisos, la confrontación o acoplamiento de prácticas conllevan valores que arrojan como resultados procesos de acomodación. Formas sintagmáticas que permiten la optimización de las mismas. Entre las estrategias — de las cuales se detallará las semejanzas y diferencias en los colegios de la muestra-, se encuentran las que vamos a mencionar a continuación.

Una primera estrategia fue encontrada en la primera sección observada del colegio católico (San José de Cluny), en el que la profesora utiliza las películas para llamar la atención de los alumnos (acá se conjuga la práctica de los alumnos de hacer ruido en clase y que la profesora no tiene mucha autoridad y un tono de voz bajo, entonces utiliza como una forma sintagmática nueva la incorporación de los videos); lo cual se asemeja a la estrategia utilizada en el colegio laico (Independencia) donde, para evitar la distracción de los alumnos, también se utiliza videos reproducidos a través de un proyector. Esta estrategia contrasta con el colegio cristiano (Jesús Salvador), en el cual no se utiliza material audiovisual; sin embargo, la profesora utiliza otro tipo de estrategias: por ejemplo, tocar temas tabú para llamar la atención de los alumnos distraídos (en el caso particular observado, los alumnos estaban distraídos por las actividades realizadas por el Día de la Madre fuera del salón; en este caso se conjuga la práctica de la distracción y ruido causado por los alumnos, con la de la profesora hablando de temas polémicos).

Una estrategia utilizada en el colegio católico (en el que la religiosa no tenía mucha autoridad) era hacer cantar y actuar a los alumnos preparándolos para su visita misionera y, en otras secciones, realizar el juego de la charada; a diferencia de las otras dos instituciones (Jesús Salvador e Independencia), donde no se usaba esta estrategia para llamar la atención de los alumnos. Otra estrategia utilizada en general por los tres colegios es usar el libro sagrado o Biblia para que, cuando los alumnos están perdiendo la concentración, lean cada uno un párrafo o respondan preguntas acerca de lo que leen.

En el caso del colegio cristiano (Jesús Salvador), encontramos estrategias particulares y únicas de esta institución. Por ejemplo, cuando la profesora amenaza con que los que no bendigan al pueblo de Israel no serán bendecidos por Dios (estrategia en donde se conjuga la práctica que tiene el colegio de bendecir a dicho pueblo todas las 
mañanas con la de algunos alumnos que se quedan callados en vez de decir la frase que les exige el colegio: "Israel bendeciré a los que te bendigan"); estrategia que lógicamente no es encontrada en ninguna de las otras dos instituciones.

Otra estrategia particular del colegio evangélico (Jesús Salvador) era la que usaba la profesora para llamar la atención de los alumnos distraídos escenificando ella misma, gesticulando y hablando muy fuerte sobre los pasajes de la Biblia que se estaban estudiando en el momento de la información; lo que difiere de los colegios laico y católico (San José de Cluny e Independencia) donde, para llamar la atención de los alumnos, los profesores prefieren apoyarse en elementos audiovisuales, como el uso del Power Point o videos.

También encontramos, entre las estrategias encontradas en el colegio evangélico (Jesús Salvador), aquella que, ante la práctica de los alumnos de hacer preguntas sobre las dudas que tienen sobre lo que dice el libro sagrado, la profesora responde que las cosas son así porque así dice la Biblia, lo que difiere de los otros dos colegios donde los profesores intentan dar algún tipo de explicación o argumentación.

Por otro lado, una estrategia particular utilizada por el colegio católico (San José de Cluny) es solicitarles a aquellos alumnos que no van muy bien en el curso de religión que asistan a la pastoral de los sábados donde serían más instruidos en el tema religioso, estrategia que no fue encontrada en ninguno de los otros dos colegios de la muestra.

Una estrategia en común encontrada en los tres colegios es el uso del sistema de puntaje para mantener a los alumnos concentrados. Ya que en los tres colegios el curso de religión es una materia calificada y cuyo promedio cuenta para el ponderado final del año, los profesores suelen amedrentar a los alumnos que interrumpen la clase o hacen ruido diciéndoles que se les bajará puntos en su nota final.

\section{c. Formas de vida}

Finalmente, a los resultados le agregamos la comparación y análisis de las formas de vida encontradas en la observación de la muestra. Se puede hablar de formas de vida cuando se identifican estilos estratégicos coherentes, recurrentes, relativamente independientes de las situaciones temáticas y suficientemente poderosas para 
influenciar todas las prácticas y todas las manifestaciones semióticas de un grupo o de un tipo social y cultural.

En el caso de las formas de vida encontradas, vemos que el colegio católico (San José de Cluny) busca difundir básicamente el conocimiento teórico de los valores católicos y del mensaje de la Biblia, mas no se entromete directamente con el modo de vida de los alumnos; a diferencia del colegio evangélico (Jesús Salvador), donde pudo verse que se inculcaba un modo de vida en los alumnos que vaya de acuerdo con los valores evangélicos enseñados por el colegio. También encontramos, como forma de vida, que el colegio evangélico busca difundir el conocimiento de la Biblia desde el punto de vista evangélico.

Las formas de vida mencionadas anteriormente difieren de aquella hallada en el colegio laico (Independencia) donde, como resultado, obtuvimos que esta consiste en cumplir con la currícula que manda el Ministerio de Educación, la cual incluye el curso de religión. Una semejanza que encontramos entre las formas de vida del colegio católico y el laico es que en el segundo, aparte de cumplir con la currícula, al impartir el curso de religión también busca difundir valores católicos en los alumnos practicantes, siendo la religión católica la que está incluida en la malla curricular establecida por el Gobierno.

Si retomamos la idea de la continuidad y discontinuidad inaugurada por Floch y la formulamos como formas de vida, podemos establecer una continuidad en los procesos semióticos activados desde la perspectiva del colegio católico y laico (San José de Cluny e Independencia) a diferencia de la no discontinuidad en el caso del colegio evangélico (Jesús Salvador). Mientras en el primero la materia del curso y todos los elementos metodológicos parecen sugerir un procedimiento casi ordenado por el estado natural de las cosas, en el caso del segundo encontramos intentos de encadenamientos entre las prácticas de enseñanza y aprendizaje para lograr integrar los contenidos religiosos a la vida cotidiana de los educandos. Mientras que en el primero el curso discurre de manera automática sin ningún intento por integrarse en las prácticas cotidianas, observamos un escenario diferente con respecto a la práctica de la educación evangélica aunque ésta tenga que valerse de amenazas. 


\section{DISCUSIÓN}

Recordemos que los objetivos de esta investigación son la identificación de prácticas, estrategias y formas de vida encontradas en los métodos de enseñanza del curso de religión en tres colegios de diferentes confesiones: católica, evangélica y laico. Para esto se recurrió a la observación no participante de tres secciones (cuarto, quinto y sexto de primaria). Entre los hallazgos principales, se encontraron las diferentes formas de vida, algunas estrategias particulares a cada colegio y ciertas prácticas semejantes, así como otras diferentes. Se planteó como hipótesis inicialmente la confluencia de diferencias entre el colegio católico y el evangélico; sin embargo, también se encontraron semejanzas. En cuanto al laico, la situación difiere un poco de lo esperado, ya que se esperaba encontrar menor relación entre la religión y el Estado. Dicha relación termina haciendo del colegio laico una versión moderada del colegio católico.

En el caso del colegio laico, podemos contrastar con el artículo científico de Nanbu Hirotaka (2008), en el que menciona cómo el régimen chino, el cual fue un adversario de la religión por mucho tiempo, finalmente terminó utilizándola a su favor. Una de las formas de vida encontradas en el análisis del colegio laico (Independencia) fue, aparte de cumplir con la currícula impuesta por el Gobierno (la cual incluye el curso de religión), que también se busca fomentar valores católicos. Se podría deducir que, dada la situación actual del país debido a la pobreza y otros problemas de índole social (alta tasa de delincuencia, abandono escolar y problemas familiares), en cierta forma, los valores católicos pueden ayudar al Gobierno en la educación moral del estudiante y en la prevención de estos problemas. No obstante, si la forma de vida hallada en la educación religiosa es la de una continuidad que deja el contenido religioso distante de la vida cotidiana esto no se produciría. Sólo maestros comprometidos con la religión que enseñan podrían hacer de su curso un eslabón preventivo a los problemas mencionados anteriormente.

Entre los objetivos que se esperaba alcanzar en la comparación entre el colegio católico y el evangélico (San José de Cluny y Jesús Salvador), en cierta forma se esperaba que las prácticas, estrategias y formas de vida fueran distintas, confirmándolo a través de la observación. En efecto, se encontraron diferencias en las prácticas, aunque algunas semejanzas, como aquella de que en ambos las prácticas responden a sus ritos 
respectivos. En el caso de las estrategias, hubo algunas semejantes, pero otras distintas, especialmente en el colegio cristiano (Jesús Salvador), que usaba estrategias muy ligadas al uso del miedo, el castigo de Dios frente a temas de índole sexual, las que se sabe son contrarias a la práctica evangélica que busca promover la institución. Llegando finalmente a las formas de vida se encontraron diferencias en ambas instituciones, es ahí donde se puede identificar más claramente la diferencia: el colegio católico (San José de Cluny) busca más que todo enseñar la parte teórica de la religión, mientras que el evangélico (Jesús Salvador) busca inculcar un modo de vida religioso a sus alumnos.

Contrastando esto con los antecedentes, se puede identificar la situación que se vive actualmente en el Perú, con la proliferación de iglesias e instituciones evangélicas, en lo mencionado en la investigación de Siebzehner y Lehmann (2008). Por casi 500 años (desde las épocas coloniales) la religión católica fue impuesta tanto en el Perú como en Sudamérica y su influencia fue muy grande hasta el año 1950, cuando empezaron a llegar las primeras iglesias evangélicas a nuestro país, hasta su boom en 1990. Recuérdese que la iglesia católica buscó por muchos años fortalecer el poder de la colonia, la segregación y las costumbres coloniales; lo cual difiere de la propuesta evangélica que tiene su mayor congregación en las zonas menos favorecidas económicamente del Perú. Se podría deducir que aquellos que se sentían en cierta forma discriminados por su raza o condición social han optado por seguir la corriente evangélica antes que la católica, al igual que lo mencionado en el artículo de Siebzehner y Lehman que hace referencia a los judíos sefardís que han creado una nueva corriente de enseñanza religiosa al sentirse discriminados por la clásica corriente de educación religiosa asquenazí. Nuestros resultados ponen en evidencia las consecuencias en la enseñanza religiosa cuando está ligada al poder o Estado, una visión teórica que no pone en cuestión el status quo ni se interesa porque ella se encarne en la vida cotidiana. La incursión evangélica no solamente es presencia de más iglesias en las zonas más pobres del país, es el planteamiento de una religión encarnada o encadenada semióticamente a la vida cotidiana con sus problemas y vicisitudes.

Los aspectos mencionados anteriormente sobre la relación entre la educación católica y la colonia se mencionan en el artículo escrito por Torrendell, en el que expone las tendencias y problemas culturales, educativos y magisteriales de la educación católica en Sudamérica, considerando aspectos tales como el monopolio o unanimidad de la educación católica en la época colonial, las tensiones entre el Estado y la 
educación católica durante las primeras décadas posteriores a las independencias de los países latinoamericanos y la consolidación del Estado educador y el crecimiento paralelo de la educación (Torrendell 2010).

Continuando con los antecedentes y los resultados obtenidos, observamos que estos se parecen bastante a lo mencionado en la investigación de Gärtner y Könemann (2013), artículo en el que se menciona cómo en el caso de Alemania, y con la ruptura y debilitamiento de las tradiciones cristianas, la forma de inculcarlas pasó a darse a través de la escuela; lo que se parece bastante al caso peruano, tanto en colegios evangélicos como en los católicos, que se vuelven el lugar para fortalecer estas tradiciones que muchas veces no se practican en la familia o la vida diaria de los alumnos. Para concluir con los objetivos y su contraste con los antecedentes, podemos decir que, tanto el objetivo general como los objetivos específicos, se cumplieron en la medida que se llegó a analizar lo que se pretendía utilizando el método semiótico que se requería para esta investigación.

Hay que resaltar que, si bien ningún método semiótico es perfecto en su totalidad, los planos de inmanencia - el modelo de Jacques Fontanille - fueron elegidos especialmente para esta investigación debido a su capacidad para delimitar la observación y obtener los resultados que, si bien eran también el análisis de prácticas y estrategias, se buscaba llegar a la forma de vida, el nivel más alto de los planos de inmanencia y aquel que puede resumir más fácilmente el análisis de la cultura o religión. Como observación a este método, se puede decir que limita el estudio al campo puramente semiótico y no permite ir más allá, a encontrar las causas de las cosas, aunque nos da pistas claras que podemos utilizar para deducirlo nosotros mismos mediante la utilización de antecedentes, nuestro propio estudio de la realidad y la bibliografía existente.

Otra pequeña crítica o limitación de este modelo es que resulta una foto del instante en que se hace la investigación, mas no arroja predicciones sobre el futuro y lo que podrían llegar a convertirse estas formas de vida encontradas. Sin embargo, una vez más, el modelo usado puramente para la investigación, sumado al estudio de antecedentes y la propia reflexión de acuerdo a la realidad social del momento, puede arrojar resultados muy precisos y coherentes. 
Como investigación, se resalta la importancia de este estudio frente a un hecho que es evidente en estos tiempos, aunque no resulte trascendente para todos: la pérdida de la vigencia de los valores católicos; los cuales, durante muchos años, fueron aplicados a la vida diaria y han ido desapareciendo, así como su transformación y aplicación a la vida actual y, de la misma manera, compararlos a los de la iglesia evangélica; la cual, si bien está en auge, es aún minoritaria y llega principalmente a los sectores menos favorecidos de la población. La curiosidad ante este hecho lleva al uso de la semiótica para el análisis de una de las fuentes principales de socialización del ser humano como es la escuela, especialmente en las secciones de cuarto, quinto y sexto de primaria, que es cuando el ser humano llega a la pubertad y empieza a decidir por sí mismo. El método semiótico ayuda mucho a delimitar el estudio y a llegar a conclusiones globales.

Sin embargo, la investigación, especialmente en el área del trabajo de campo, presentó muchas dificultades. Como se señaló anteriormente, se utilizó el método de observación no participante dentro de las aulas para obtener información. El ingreso a estas no fue conseguido fácilmente. Es claro que ningún docente se siente cómodo cuando es informado de que alguien va a observar su clase, ya que se puede tratar de un supervisor o alguien que lo calificará como maestro. La docencia es una profesión que en el Perú ha sido muy devaluada y donde muchos maestros han sido acusados de promover ideas subversivas, por lo que el acceso a las aulas se hace difícil en algunos casos. Pudimos percibir esto especialmente en el colegio laico (Independencia), donde se encontró una fuerte resistencia de los docentes a pesar de tener el permiso de la directora; y en el colegio católico (San José de Cluny), donde la directora se negó inicialmente a dar el permiso para la observación hasta que se convenció de que la investigación no afectaría ni a los docentes ni a la reputación del colegio.

Una de las cosas más complicadas fue obtener registro fotográfico, ya que los profesores y autoridades escolares se oponían a ello, por lo menos durante la observación de la clase. De modo que, para no interferir con el dictado de clases, la posición del observador siempre fue en una carpeta al fondo del salón y con la utilización de una grabadora de sonido y una libreta de anotaciones. Por supuesto que se hubiera podido llegar más lejos en la investigación con la colaboración plena de las autoridades y profesores del colegio, así como con la utilización de la fotografía de algunas cosas, lo que lamentablemente no fue posible. 
Finalmente, como reflexión propia del investigador, se ha observado que, tanto la educación católica como evangélica en los colegios analizados, forman parte de lo que se puede denominar "educación cristiana" en general. Si bien ambas confesiones profesan ritos distintos y en sus métodos de enseñanza son diferentes, al igual que sus formas de vida en el análisis semiótico, se ha podido notar que ambas buscan fomentar los valores que pertenecen a las religiones judeo-cristianas que figuran en la Biblia. Sin embargo, se puede notar la búsqueda por influir en el modo de vida de los alumnos, en el caso del colegio cristiano (Jesús Salvador); mientras que el católico (San José de Cluny) busca influir más sobre el conocimiento teórico o en las mentes del alumnado. Además, el colegio laico (Independencia) hace notar cómo aun en estos tiempos el gobierno permanece de alguna manera ligado a la iglesia católica al ser esta la religión que promueve en su currícula.

Como conclusión, diría que la influencia en los alumnos del colegio evangélico (Jesús Salvador) responde a una búsqueda por promover su conversión y ganar más adeptos, especialmente en los padres que quieren proteger a sus hijos de los flagelos actuales de la sociedad (como el alcohol y las drogas), así como aquellos que poseen una visión conservadora o los valores católicos devaluados en la actualidad, quienes puede que vean en los colegios evangélicos la única manera de mantener estos valores.

Después de realizar esta investigación, se sugiere a futuros investigadores interesados en el tema realizar (utilizando este u otro método semiótico) un análisis de la enseñanza de religión en los colegios enfocándose a su clase social. Limitaría la investigación a una sola confesión y qué prácticas, estrategias y formas de vida son halladas en colegios católicos del sector socioeconómico A y B y cuales otras en el C, D y E. Además, sería necesario comparar y analizar los hallazgos para encontrar coherencia o incongruencia en los métodos de enseñanza y la clase social de los alumnos. Otra investigación que podemos sugerir es aquella que analice, mediante el método semiótico o sociológico, la influencia de las iglesias evangélicas en las zonas rurales y sus diferencias. Si se tratara desde el método semiótico, podrían analizarse las diferencias entre las formas de vida de la enseñanza en colegios evangélicos de zonas poco favorecidas económicamente de provincia y en Lima, para discernir y poder llegar a conclusiones que esclarezcan el porqué del crecimiento de la iglesia evangélica en el Perú. 


\section{REFERENCIAS}

Alberts, W. (2010). The academic study of religions and integrative religious education in Europe, Noruega. British Journal of Religious Education, $32(2)$

Anguera. (1997). Metodología de las ciencias humanas. Cátedra. Madrid

Floch, JM. (1993). Semiótica, Marketing y Comunicaciones: bajo los signos y estrategias. Paidós. Barcelona

Fontanille, J. (2014). Prácticas Semióticas. Universidad de Lima.

Fontanille, J. (2013). Medios, regímenes de creencia y formas de vida. Contratexto. Universidad de Lima.

Gartner y Konemann. (2013). Religion and All-Day Schools: Impact of All-Day Schools on the Systems of School and Religion, Alemania. Journal of Empirical Theology, 26 (1)

Landowski, E. (2009). Interacciones arriesgadas. Universidad de Lima. Perú

Nanbu, H. (2008). Religion in Chinese education: from denial to cooperation, China. British Journal of religious education, 30-(3)

Pardinas. (1978). Metodología y técnicas de investigación en ciencias sociales. México D.F.

Schwadel, P. (2011). The Effects of Education on Americans' Religious Practices, Beliefs, and Affiliations, USA. Review of Religious Research, 53(2)

Segall, A y Burke, K. (2013). Reading the Bible as a Pedagogical Text: Testing, Testament, and Some Postmodern Considerations About Religion/the Bible in Contemporary Education. USA. Curriculum Inquiry, 43 (3)

Sharma, S y Guest, M. (2013). Navigating religion between university and home: Christian students' experiences in English universities, Inglaterra. Social \& Cultural Geography, (14) 1. 
Schwadel, P. (2011). The Effects of Education on Americans' Religious Practices, Beliefs, and Affiliations, USA. Review of Religious Research, 53(2)

Siebzehner, B y Lehmann, D. (2008). Embracing Segregation: The Rise Of A Religious Educational System In Israel (SHAS), Israel. British Journal of religious education, 32-(33).

Teece, G. (2010). Is it learning about and from religions, religion or religious education? And is it any wonder some teachers don't get it?, Inglaterra. British Journal of Religious Education, 32(3)

Torrendell, C. (2010). Desafíos de fidelidad y caridad en la educación católica sudamericana, Argentina. Humanitas Revista de antropología cristiana, 15(58) 